Comptes Rendus Physique 8 (2007) 579-590

\title{
Network and thermodynamic conditions for a single macroscopic current fluctuation theorem
}

\author{
David Andrieux, Pierre Gaspard \\ Center for Nonlinear Phenomena and Complex Systems, \\ Université Libre de Bruxelles, Code Postal 231, Campus Plaine, B-1050 Brussels, Belgium
}

\begin{abstract}
We analyse the different situations where a fluctuation theorem can be proved for a single macroscopic current of a system in a nonequilibrium steady state sustaining several transport processes. We express the conditions under which such a fluctuation theorem will hold in terms of the cycles and transport processes of the network. In this case, a given macroscopic current obeys a fluctuation theorem regardless of the other transport processes.

\section{Résumé}

Conditions thermodynamique et de réseau du théorème de fluctuations pour un seul courant macroscopique. Nous analysons les différentes situations où un théorème de fluctuations peut être démontré pour un seul courant macroscopique d'un système dans un état stationnaire de non-équilibre soutenant plusieurs processus de transport. Nous exprimons les conditions sous lesquelles un tel théorème de fluctuations tiendra en termes des cycles et des processus de transport sur le réseau. Dans ce cas, un courant macroscopique donné obéit à un théorème de fluctuations sans implication des autres processus de transport.
\end{abstract}

Key words: Nonequilibrium steady state; entropy production ; current fluctuations ; thermodynamic forces ; fluctuation theorem. Mots-clés : Etat stationnaire de non-équilibre; production d'entropie; fluctuations de courant; forces thermodynamiques ; théorème de fluctuations.

\section{Introduction}

Natural systems are driven out of equilibrium if their temperature, pressure, or chemical potentials are non uniform. This is the feature of many physical, chemical, and biological systems $[1,2,3,4,5,6,7,8]$. Such nonequilibrium systems are crossed by currents of energy or matter generated by the aforementioned non-uniformities, also called affinities [9]. The currents contribute to positive entropy production according to the second law of thermodynamics.

At the mesoscopic level, the time evolution of these systems is ruled by a stochastic process which obeys a master equation for the probability distribution of the coarse-grained states. Accordingly, all systems present fluctuations in their currents. Recently, remarkable properties of the molecular fluctuations have been discovered

Email addresses: david.andrieux@ulb.ac.be (David Andrieux), gaspard@ulb.ac.be (Pierre Gaspard). 
in nonequilibrium systems. The time evolution of the molecular fluctuations can be characterized in terms of their large deviations with respect to their average. Several large-deviation dynamical relationships have been obtained such as the formulas of the escape-rate and hydrodynamic-mode theories [10], as well as the nonequilibrium work theorem $[11,12]$ and the fluctuation theorems $[13,14,15,16,17,18,19,20,21,22,23,24,25]$. The fluctuation theorems are symmetry relations originating from microreversibility for the probability distributions of fluctuating quantities, which are of interest for nonequilibrium thermodynamics and transport properties. These new large-deviation relationships apply to mesoscopic devices ranging from electronic transport in mesoscopic conductors [26] to chemically driven molecular motors [27]. These devices typically operate in far-from-equilibrium regimes $[28,29]$.

Here, we are interested in the fluctuation theorem for a single macroscopic current. Indeed, it has been proved in Refs. $[30,31]$ that there exists a fluctuation relation for the ensemble of nonequilibrium currents crossing the system in a nonequilibrium stationary state. It is expressed in terms of the corresponding macroscopic affinities. However, in experimental situations, it is not always possible to consider simultaneously all the currents and one usually measures a subset of all possible currents. Moreover, the question whether a fluctuation theorem exists for a single macroscopic current has been raised in relation with the sign of currents in ratchets [37]. Our aim is thus to derive the conditions under which a fluctuation relation for such a single macroscopic current will hold.

The paper is organized as follows. We introduce the stochastic description in terms of the master equation in Section 2, as well as the cycle formalism developed in Ref. [33]. In Section 3, we define the generating function of the currents and a fluctuation theorem for the cycle currents is proved at the mesoscopic level of description. We discuss the relations between the macroscopic thermodynamic quantities and the cyclic paths of the process in Section 4. In particular, we show that a single macroscopic current can be expressed in terms of the cyclic paths of the process. We then derive the sufficient and necessary conditions under which a single macroscopic current will satisfy a fluctuation symmetry. We also detail its implications on the response theory. In Section 5, we illustrate and discuss the different results for a specific Markov process and a chemical reaction network process. Conclusions are drawn in Section 6.

\section{Master equation and its cycle representation}

\subsection{The master equation}

Many nonequilibrium phenomena are successfully described at the mesoscopic level in terms of continuous-time random processes. They are ruled by an evolution equation, called the master equation, for the probability $P(\omega, t)$ to find the system in some coarse-grained state $\omega$ at time $t$ :

$$
\frac{d P(\omega, t)}{d t}=\sum_{\omega^{\prime}}\left[W\left(\omega^{\prime}, \omega\right) P\left(\omega^{\prime}, t\right)-W\left(\omega, \omega^{\prime}\right) P(\omega, t)\right]
$$

The coefficients $W\left(\omega^{\prime}, \omega\right)$ denote the rates of the transitions $\omega^{\prime} \rightarrow \omega$. We assume that, if $W\left(\omega^{\prime}, \omega\right)>0$, the reversed transition is also possible $W\left(\omega, \omega^{\prime}\right)>0$. In the general case, $W\left(\omega^{\prime}, \omega\right)$ is composed of several transition rates $W_{\rho}\left(\omega^{\prime}, \omega\right)$ corresponding to the elementary processes $\rho=1,2, \ldots, r$ and such that $W\left(\omega^{\prime}, \omega\right)=\sum_{\rho} W_{\rho}\left(\omega^{\prime}, \omega\right)$. This is for instance the case in chemical reaction networks where each reaction defines an independent macroscopic current and contributes separately to the entropy production $[6,7,13,24,30]$ so that it is important to separate them in the master equation. However, in order to simplify the notations in this paper, we here consider the case where there is only one elementary process. Its extension to the general case is straightforward.

The master equation is known to evolve towards a steady state where $d P / d t=0$. This stationary state is the equilibrium one if the conditions of detailed balance

$$
P_{\mathrm{eq}}(\omega) W\left(\omega, \omega^{\prime}\right)=P_{\mathrm{eq}}\left(\omega^{\prime}\right) W\left(\omega^{\prime}, \omega\right)
$$

are satisfied for all the possible forward and backward transitions $\omega \rightleftharpoons \omega^{\prime}$. An equivalent condition can be expressed in terms of the sole transition rates as

$$
W\left(\omega_{1}, \omega_{2}\right) W\left(\omega_{2}, \omega_{3}\right) \cdots W\left(\omega_{n}, \omega_{1}\right)=W\left(\omega_{1}, \omega_{n}\right) W\left(\omega_{n}, \omega_{n-1}\right) \cdots W\left(\omega_{2}, \omega_{1}\right)
$$

for every cyclic path $\omega_{1} \rightarrow \omega_{2} \rightarrow \cdots \rightarrow \omega_{n} \rightarrow \omega_{1}$ [7]. In the following, such a cycle will be denoted by $c \equiv$ $\left(\omega_{1} \omega_{2} \cdots \omega_{n}\right)$ and the corresponding reversed cycles by $c_{-} \equiv\left(\omega_{1} \omega_{n} \cdots \omega_{2}\right)$. Here, we introduce the quantities $w_{c} \equiv$ 
$W\left(\omega_{1}, \omega_{2}\right) \cdots W\left(\omega_{n-1}, \omega_{n}\right) W\left(\omega_{n}, \omega_{1}\right)$ as the product of the transition rates along the cycle $c$. The equilibrium conditions (3) are thus equivalent to

$$
w_{c}=w_{c_{-}}
$$

for every cycle $c$. If this condition is violated for a cycle, the system is in a nonequilibrium stationary state characterized by the presence of non vanishing probability currents and a positive entropy production [7].

\subsection{Stochastic trajectories and cycle representation}

The equilibrium and nonequilibrium properties of the master equation can be characterized by a cycle representation of the Markov process. Such representation of the master equation has been extensively used in the biochemical context by Hill $[34,35,36]$. This approach was latter developed by the Qians $[13,33]$. We now introduce this framework in detail as we use this description in the following sections.

A stochastic trajectory $\underline{\omega}$ corresponding to the master equation (1) is composed of transitions $e_{n} \equiv \omega_{n-1} \rightarrow \omega_{n}$ at random times $t_{n}$. These transitions are the directed edges of the graph of the process while the states $\omega_{n}$ are its vertices. In this respect, we can consider a derived process $\eta$ where we prune and list the cycles formed up to time $t$. An example of construction of this process is

\begin{tabular}{c|ccccccccc}
$n$ & 0 & 1 & 2 & 3 & 4 & 5 & 6 & 7 & $\ldots$ \\
$\omega_{n}$ & 1 & 2 & 3 & 4 & 2 & 3 & 5 & 1 & $\ldots$ \\
$\eta_{n}$ & {$[1]$} & {$[12]$} & {$[123]$} & {$[1234]$} & {$[12]$} & {$[123]$} & {$[1235]$} & {$[1]$} & $\ldots$ \\
cycles formed & - & - & - & $(234)$ & - & - & $(1235)$ & $\ldots$
\end{tabular}

where $\omega_{n}$ denotes the states of the original process $\underline{\omega}$ and $\eta_{n}$ the states of the derived process $\eta$. We use the notations of Ref. [13] where the process $\eta$ has been extensively studied.

In this representation, all the different cycles $c$ are taken into account at the time each cycle is completed. A graphical representation of the derived process $\eta$ is given by a tree with vertices corresponding to states $\left[\omega_{0} \omega_{1} \cdots \omega_{s}\right]$. The states of the process $\eta$ will be denoted $\eta$ in the following. We notice that there is usually much more states $\eta$ than original states $\omega$. However, this is not exactly a tree since there exist transitions towards the previous states. In this case, one necessarily accomplishes the formation of a cycle and this transition is irreversible (except in the case of back and forth cycles $\left.\left[\omega_{0} \omega_{1} \cdots \omega_{s}\right] \rightarrow\left[\omega_{0} \omega_{1} \cdots \omega_{s} \omega_{s+1}\right] \rightarrow\left[\omega_{0} \omega_{1} \cdots \omega_{s}\right]\right)$. Indeed, in the previous example, we see that the transition [1234] $\rightarrow[12]$ can be accomplished but not the reversed one. An important point is that the properties of a state $\left[\omega_{0} \omega_{1} \cdots \omega_{s}\right]$ are uniquely determined by its final value $\omega_{s}$ when considering the transition rates and waiting times. We thus define a mapping $\tilde{\eta}=\left[\omega_{0} \omega_{1} \cdots \omega_{s}\right] \rightarrow \omega_{s}$. Another important remark is that the process $\eta$ constructed in this way depends on the initial value $\omega_{0}$ of the trajectory. However, it has been shown in Ref. [13] that each such subprocess presents the same long-time properties.

We can now assign an arbitrary orientation to every different cycle so that it splits into a forward cycle $c_{+}$ and a backward cycle $c_{-}$. The associated current $j_{c}$ is positive when the cycle $c_{+}$is completed and negative when the cycle $c_{-}$is. The associated cycle currents are thus defined as $j_{c}=j_{c_{+}}-j_{c_{-}}$along with their mean values $J_{c_{ \pm}} \equiv\left\langle j_{c_{ \pm}}\right\rangle$. We notice that the cycle currents $j_{c}$ are time-dependent fluctuating quantities while their mean values $J_{c}=\left\langle j_{c}\right\rangle$ are stationary if the system is in a steady state. The currents in the positive and negative orientations are related by $J_{c_{+}} / J_{c_{-}}=\exp \left(w_{c_{+}} / w_{c_{-}}\right)$as shown in $[13,36]$. It is therefore natural to define the affinity of a cycle as

$$
A(c)=\ln \frac{w_{c_{+}}}{w_{c_{-}}}
$$

This is also consistent with Schnakenberg's approach [7]. 


\section{Fluctuation theorem for mesoscopic cycle currents}

In this section we want to show that one can derive a fluctuation theorem for single cycle currents as introduced in Section 2. We point out that these cycle currents are still mesoscopic properties.

We consider the derived process $\underline{\eta}$. We associate with each different cycle $c_{ \pm}$a corresponding parameter $\lambda_{c}$. The generating function is defined as

$$
Q\left(\lambda_{c_{1}}, \lambda_{c_{2}}, \ldots, \lambda_{c_{r}}\right) \equiv \lim _{t \rightarrow \infty}-\frac{1}{t} \ln \left\langle\mathrm{e}^{-\sum_{c} \lambda_{c} \int_{0}^{t} d t^{\prime} j_{c}\left(t^{\prime}\right)}\right\rangle
$$

We first show that the generating function $Q$ presents the symmetry

$$
Q\left(\lambda_{c_{1}}, \ldots, \lambda_{c_{s}}, \ldots, \lambda_{c_{r}}\right)=Q\left[\lambda_{c_{1}}, \ldots, A\left(c_{s}\right)-\lambda_{c_{s}}, \ldots, \lambda_{c_{r}}\right]
$$

for any cycle $c_{s}$ and this in terms of its corresponding affinity (5). This remarkable result is a consequence of the special structure of the derived process $\eta$. In particular, this result shows that all the cycles are independent in the long-time limit.

The generating function (6) adds a contribution $\exp \left( \pm \lambda_{c}\right)$ each time a directed cycle $c_{ \pm}$is completed. In order to simplify the proof without loss of generality, we will consider the process $\eta$ in discrete time.

This is not a restriction since we are here interested in a topological property holding in the long-time limit so that it is equivalent to consider the embedded Markov chain defined by $p_{\omega \omega^{\prime}}=W\left(\omega, \omega^{\prime}\right) / \sum_{\omega^{\prime}} W\left(\omega, \omega^{\prime}\right)$ if $\omega \neq \omega^{\prime}$ and $p_{\omega \omega}=0$ otherwise. In this case, the probability to observe a trajectory $\underline{\omega}=\omega_{1} \cdots \omega_{n}$ is given by

$$
P(\underline{\omega})=P\left(\omega_{1} \cdots \omega_{n}\right)=p_{\mathrm{st}}\left(\omega_{1}\right) p_{\omega_{1} \omega_{2}} \cdots p_{\omega_{n-1} \omega_{n}}
$$

If this trajectory $\underline{\omega}$ contains $n_{c}$ cycles of type $c$, the corresponding trajectory $\underline{\omega}^{\prime}$ where all the cycles of type $c_{s}$ are completed in the reverse direction has the probability

$$
P\left(\underline{\omega}^{\prime}\right)=P(\underline{\omega}) \exp \left[-n_{c_{s}}(\underline{\omega}) A\left(c_{s}\right)\right]
$$

where we used Eq. (5). We notice that this property would not hold for the process $\underline{\omega}$, in which the currents do not change at the moments the cycles are completed. The probability $P\left(N_{c_{1}}, \cdots, N_{c_{s}}, \cdots, N_{c_{r}}\right)$ to observe a fixed number $N_{c_{i}}$ of cycles is given by the sum of the probabilities of all the trajectories satisfying the constraints $\left\{N_{c_{i}}\right\}$ so that

$$
P\left(N_{c_{1}}, \cdots,-N_{c_{s}}, \cdots, N_{c_{r}}\right)=P\left(N_{c_{1}}, \cdots, N_{c_{s}}, \cdots, N_{c_{r}}\right) \exp \left[-N_{c_{s}} A\left(c_{s}\right)\right]
$$

since the relation (9) is valid for every trajectory. Therefore

$$
\begin{aligned}
\left\langle\mathrm{e}^{-\sum_{i} \lambda_{c_{i}} N_{c_{i}}}\right\rangle & =\sum_{N_{c_{i}}=-\infty}^{+\infty} P\left(N_{c_{1}}, \cdots, N_{c_{s}}, \cdots, N_{c_{r}}\right) \mathrm{e}^{-\sum_{i} \lambda_{c_{i}} N_{c_{i}}} \\
& =\sum_{N_{c_{i}}=-\infty}^{+\infty} P\left(N_{c_{1}}, \cdots,-N_{c_{s}}, \cdots, N_{c_{r}}\right) \mathrm{e}^{-\left[\lambda_{c_{s}}-A\left(c_{s}\right)\right] N_{c_{s}}} \mathrm{e}^{-\sum_{i \neq s} \lambda_{c_{i}} N_{c_{i}}} \\
& =\left\langle\mathrm{e}^{-\left[A\left(c_{s}\right)-\lambda_{c_{s}}\right] N_{c_{s}}} \mathrm{e}^{-\sum_{i \neq s} \lambda_{c_{i}} N_{c_{i}}}\right\rangle
\end{aligned}
$$

hence Eq. (7). This argument explicitly shows that the result (7) is based on the definition that the current $j_{c}$ changes at the moment the cycle $c$ is completed. The result (7) would not hold otherwise. Therefore, we have proved the symmetry relation $(7)$.

\section{Conditions for single macroscopic current fluctuation theorems}

In this section, we will consider the fluctuations of a single macroscopic current. A fluctuation theorem for the ensemble of macroscopic currents has been derived in Refs. [30,31]. We will use the results of the previous section in order to derive the general conditions under which a given macroscopic current will also satisfy a fluctuation symmetry. 


\subsection{Thermodynamic description}

We first show how thermodynamic quantities like macroscopic currents and affinities can be expressed in terms of the cycles of the process. Indeed, from a macroscopic point of view, there is a finite number of irreversible processes $k$. Each one is characterized by a mean current $J_{k}$ and a driving force or affinity $X_{k}$ [1]. Schnakenberg showed how to extract these independent quantities in the stochastic description [7] thanks to a graphical representation of the master equation. In this framework, a maximal tree defines a set of independent cycles. A set of independent affinities $X_{k}$ can be associated with these fundamental cycles and they correspond to the macroscopic affinities defined in terms of the differences of temperature, pressure, or chemical potentials between the heat or particle reservoirs maintaining the system out of equilibrium.

Each cycle in the graph can be expressed in terms of the set of fundamental cycles [7]. The affinity $A(c)$ of a cycle $c$ can thus be expressed in terms of the independent affinities $X_{k}$ of the system [7] as

$$
A(c)=\sum_{k} \rho_{k}(c) X_{k}
$$

where $\rho_{k}(c) \in \mathbb{Z}$ counts the (positive or negative integer) contribution of the cycle to the given transport process $k$ and can be obtained from the structure of the graph [7]. Accordingly, a cycle $c$ will contribute to the macroscopic current $J_{k}$ with a factor $\rho_{k}(c)$ so that [13]

$$
J_{k}=\sum_{c} \rho_{k}(c) J_{c}
$$

This will be illustrated by an example in Section 5 .

\subsection{Single macroscopic current fluctuation theorems}

We now consider the fluctuations of a particular macroscopic current $i$. If a cycle $c$ contributes to the macroscopic current $i$ by a factor $\rho_{i}(c)$ then the generating function of the current $i$ should thus be defined as

$$
\bar{Q}_{i}(\lambda) \equiv \lim _{t \rightarrow \infty}-\frac{1}{t} \ln \left\langle\mathrm{e}^{-\lambda \sum_{c} \rho_{i}(c) \int_{0}^{t} d t^{\prime} j_{c}\left(t^{\prime}\right)}\right\rangle
$$

It is expressed in terms of the generating function of the cycles (6) as

$$
\bar{Q}_{i}(\lambda)=Q\left[\rho_{i}\left(c_{1}\right) \lambda, \cdots, \rho_{i}\left(c_{r}\right) \lambda\right] .
$$

A first possibility is that only one cycle $c_{s}$ contributes to the macroscopic current $J_{i}$, so that $\rho_{i}\left(c_{l}\right)=0$ for $c_{l} \neq c_{s}$. In this case, the symmetry (7) directly leads to $\bar{Q}_{i}(\lambda)=\bar{Q}_{i}\left[A\left(c_{s}\right) / \rho_{i}\left(c_{s}\right)-\lambda\right]$.

In the general case where several cycles contribute to the macroscopic current $J_{i}$, we will look at the evolution operator $\hat{L}$ of the generating function and its eigenvalues $s$ as done in Ref. [31] but at the level of the process $\eta$. The matrix $\hat{L}$ has thus elements $L_{\eta \eta^{\prime}}=W_{\omega \omega^{\prime}}$ for transitions of type $\eta=\left[\omega_{0} \cdots \omega\right] \rightarrow \eta^{\prime}=\left[\omega_{0} \cdots \omega \omega^{\prime}\right]$ and elements $L_{\eta \eta^{\prime}}=W_{\omega \omega^{\prime}} \exp \left[-\lambda \rho_{i}(c)\right]$ if the transition $\eta \rightarrow \eta^{\prime}$ corresponds to the completion of cycle $c$ in the $\omega$ space. Its diagonal elements are $-\sum_{j} W_{i j}$. The eigenvalues equation thus reads

$$
\operatorname{det}(\hat{L}-s \hat{I})=\operatorname{det} \hat{M}=0
$$

where we have introduced the matrix $\hat{M} \equiv \hat{L}-s \hat{I}$. The eigenvalues $s$ are thus obtained as the zeroes of a polynomial whose coefficients are functions of the parameter $\lambda$. The determinant can also be expressed as a sum over the permutation group. The contribution of a cycle $c$ to the determinant of the operator $\hat{M}$ is therefore expressed as $m_{c}=M_{\eta_{0} \eta_{1}} M_{\eta_{1} \eta_{2}} \cdots M_{\eta_{n} \eta_{0}}=w_{c} \exp \left[-\rho_{i}(c) \lambda\right] \times D(c)$. Here, $D(c)$ is the determinant of $\hat{M}$ with the columns and lines indexed in the complementary set of $c=\left(\omega_{1} \cdots \omega_{m}\right)$ and where we have also removed all the other cyclic contributions. In particular, we note that $D\left(c_{+}\right)=D\left(c_{-}\right)=D(c)$. Likewise, we can define the quantities $D\left(c_{1}, \ldots, c_{r}\right)$. The dependence on the eigenvalues $s$ is contained in the terms $D\left(c_{1}, \ldots, c_{r}\right)$. The determinant is then expressed as a sum over all possible combinations of cycles.

Therefore, let us consider the case where we have at least two cycles $c_{1}$ and $c_{2}$ contributing to $J_{i}$, that is with $\rho_{i}\left(c_{1}\right), \rho_{i}\left(c_{2}\right) \neq 0$. In this case, they are either disjoint (i.e. they do not possess any common edge) or not. In the case the cycles $c_{1}$ and $c_{2}$ are disjoint, this determinant contains the terms 


$$
\begin{aligned}
& {\left[\mathrm{e}^{-\lambda \rho_{i}\left(c_{1+}\right)}+\mathrm{e}^{\lambda \rho_{i}\left(c_{1+}\right)-A\left(c_{1}\right)}\right] w_{c_{1+}} D\left(c_{1}\right)} \\
& +\left[\mathrm{e}^{-\lambda \rho_{i}\left(c_{2+}\right)}+\mathrm{e}^{\lambda \rho_{i}\left(c_{2+}\right)-A\left(c_{2}\right)}\right] w_{c_{2+}} D\left(c_{2}\right) \\
& +\left\{\mathrm{e}^{-\lambda\left[\rho_{i}\left(c_{1+}\right)+\rho_{i}\left(c_{2+}\right)\right]}+\mathrm{e}^{\lambda\left[\rho_{i}\left(c_{1+}\right)+\rho_{i}\left(c_{2+}\right)\right]} \mathrm{e}^{-A\left(c_{1}\right)-A\left(c_{2}\right)}\right\} w_{c_{1+}} w_{c_{2+}} D\left(c_{1}, c_{2}\right) \\
& +\cdots
\end{aligned}
$$

where we used that $\rho_{i}\left(c_{+}\right)=-\rho_{i}\left(c_{-}\right)$, as well as the definition of the affinities (5). On the other hand, any symmetry of the type $\lambda \rightarrow A-\lambda$ must be satisfied for any power of $s$. This implies that there cannot be any such symmetry unless we have $A=A\left(c_{1}\right) / \rho_{i}\left(c_{1}\right)=A\left(c_{2}\right) / \rho_{i}\left(c_{2}\right)=\cdots=A\left(c_{s}\right) / \rho_{i}\left(c_{s}\right)$ for all the cycles with $\rho_{i}\left(c_{s}\right) \neq 0$.

In the case the cycles $c_{1}$ and $c_{2}$ have a common vertex, we have instead

$$
\begin{aligned}
& {\left[\mathrm{e}^{-\lambda \rho_{i}\left(c_{1+}\right)}+\mathrm{e}^{\lambda \rho_{i}\left(c_{1+}\right)-A\left(c_{1}\right)}\right] w_{c_{1+}} D\left(c_{1}\right)} \\
& +\left[\mathrm{e}^{-\lambda \rho_{i}\left(c_{1+}\right)}+\mathrm{e}^{\lambda \rho_{i}\left(c_{2+}\right)-A\left(c_{2}\right)}\right] w_{c_{2+}} D\left(c_{2}\right) \\
& +\cdots
\end{aligned}
$$

Since each term of the determinant $\operatorname{det} \hat{M}$ corresponds to a permutation of the states, a term corresponding to a configuration with a state common to both cycles $c_{1}$ and $c_{2}$ is not possible. Indeed, such a configuration would contradict the property of invertibility of the permutation group. Therefore, the term containing both cycles $c_{1}$ and $c_{2}$ does not appear in this case. Consequently, we here also see that there cannot be any symmetry of the type $\lambda \rightarrow A-\lambda$ if the conditions $A=A\left(c_{1}\right) / \rho_{i}\left(c_{1}\right)=A\left(c_{2}\right) / \rho_{i}\left(c_{2}\right)=\cdots=A\left(c_{s}\right) / \rho_{i}\left(c_{s}\right)$ for all the cycles with $\rho_{i}\left(c_{s}\right) \neq 0$ are not satisfied. In the case $\rho_{i}\left(c_{1+}\right)=\rho_{i}\left(c_{2+}\right)$, we cannot have a more complex symmetry because the terms $D(c)$ still contains the eigenvalues $s$ and any symmetry should be satisfied for any power of $s$.

We thus have shown that the constraints derived above are necessary conditions in order for a single macroscopic current symmetry to hold.

We can now use the result (7) to show that they also define sufficient conditions. Indeed, if the conditions $A=A\left(c_{1}\right) / \rho_{i}\left(c_{1}\right)=A\left(c_{2}\right) / \rho_{i}\left(c_{2}\right)=\cdots=A\left(c_{s}\right) / \rho_{i}\left(c_{s}\right)$ for all the cycles with $\rho_{i}\left(c_{s}\right) \neq 0$ are satisfied, the correspondence (15) and the symmetry $(7)$ immediately imply $\bar{Q}(\lambda)=\bar{Q}(A-\lambda)$.

We now address the problem of determining the constant $A$. Let us consider the fundamental cycle $C_{i}$ defining the current $J_{i}$ [7]. In particular, it satisfies $\rho_{i}\left(C_{i}\right)=1$ as well as $A\left(C_{i}\right)=X_{i}$. The constant $A$ is thus simply given by $X_{i}$.

We therefore obtain our central result:

Fluctuation theorem for a single macroscopic current: The generating function (14) of the integrated fluctuating current obeys the symmetry

$$
\bar{Q}_{i}(\lambda)=\bar{Q}_{i}\left(X_{i}-\lambda\right)
$$

if and only if the network is such that $X_{i}=A\left(c_{1}\right) / \rho_{i}\left(c_{1}\right)=A\left(c_{2}\right) / \rho_{i}\left(c_{2}\right)=\cdots=A\left(c_{s}\right) / \rho_{i}\left(c_{s}\right)$ for all the cycles $c$ with $\rho_{i}(c) \neq 0$.

Let us consider in more detail the conditions of the theorem. According to Eq. (12), the affinity of a cycle will contain the term $\rho_{i}(c) X_{i}$ so that the constant ratio $A\left(c_{l}\right) / \rho_{i}\left(c_{l}\right)=X_{i}$ imposes

$$
0=\sum_{j \neq i} \rho_{j}\left(c_{l}\right) X_{j}
$$

for the cycles $c_{l}$ such that $\rho_{i}\left(c_{l}\right) \neq 0$. The usual situation where these conditions (20) are satisfied is when the other transport processes contributing to the given current $i$ are partially at equilibrium.

We can also consider the more complex case with several mechanisms $\rho$ of transitions between the states, as discussed in Section 2. The main reasoning remains unchanged. However, we will show in the next section that, in some specific cases, one can nevertheless satisfy an individual fluctuation symmetry even if the conditions $A=A\left(c_{1}\right) / \rho_{i}\left(c_{1}\right)=A\left(c_{2}\right) / \rho_{i}\left(c_{2}\right)=\cdots=A\left(c_{s}\right) / \rho_{i}\left(c_{s}\right)$ for all the cycles with $\rho_{i}(c) \neq 0$ are not met. Indeed, the conditions derived in the present section are sufficient but not necessary conditions when there exists several transition mechanisms $\rho$. 


\subsection{Consequences}

It is important to notice that the fluctuation symmetry (19) does not depend explicitly on the other affinities present in the system. This is an important point regarding the theory of linear and nonlinear responses. Indeed, one can use the fluctuation theorem for the set of currents to derive relations between the response coefficients and the fluctuations in near- and far-from-equilibrium situations [32]. Here, we demonstrated that, under certain circumstances, a single macroscopic current presents a symmetry involving only one affinity. We may wonder about the implications for the response theory. The mean currents can be expanded in terms of the macroscopic affinities as

$$
J_{\alpha}=\sum_{\beta} L_{\alpha \beta} X_{\beta}+\frac{1}{2} \sum_{\beta, \gamma} M_{\alpha \beta \gamma} X_{\beta} X_{\gamma}+\frac{1}{6} \sum_{\beta, \gamma, \delta} N_{\alpha \beta \gamma \delta} X_{\beta} X_{\gamma} X_{\delta}+\cdots+\frac{1}{n !} \sum_{\beta, \cdots, \mu} C_{\alpha \beta \cdots \mu}^{(n)} \underbrace{X_{\beta} \ldots X_{\mu}}_{n}+\cdots
$$

We now consider the response with respect to the set of thermodynamic forces that are not equal to zero for the fluctuation theorem (19) to hold. Using the symmetry (19) and the response theory of Ref. [32], we see that all the coefficients $C_{\alpha \beta \cdots \mu}^{(n)}$ are equal to zero if all the indices $\beta, \cdots, \mu$ are different from $\alpha$. All the remaining contributions are thus at least proportional to $X_{\alpha}$. In particular, the Onsager reciprocity relations are trivially satisfied since $L_{\alpha \beta}=0$ if $\beta \neq \alpha$. Thus, the mean current has the form

$$
J_{\alpha}=\Gamma^{(1)} X_{\alpha}+\frac{1}{2} \Gamma^{(2)} X_{\alpha}^{2}+\frac{1}{6} \Gamma^{(3)} X_{\alpha}^{3}+\cdots
$$

with coefficients $\Gamma^{(n)}$ depending on the other affinities $X_{\beta}$ with $\beta \neq \alpha$. Therefore, the mean current vanishes if and only if $X_{\alpha}=0$ regardless of the other thermodynamic processes at stake.

\section{Examples}

\subsection{Model with seven states}

In this section, we illustrate the result of Section 3. We consider a schematic Markov process with 7 states. The graph of this Markovian process is depicted in Fig. 1.

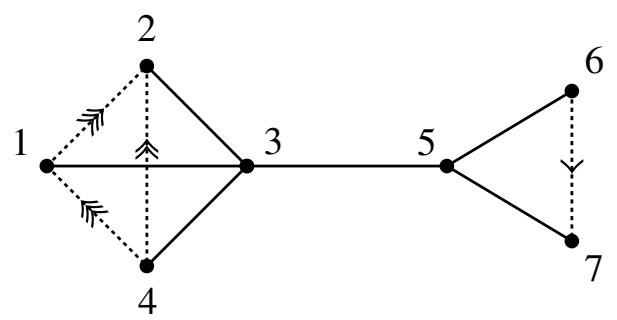

Figure 1. Graph of the Markov process with 7 states. There exist 8 cycles in the graph which are specified in the text. The currents are defined on the dashed edges which are the chords of the four independent currents. The chords $1 \rightarrow 2$ and $4 \rightarrow 1$ are supposed to contribute equally to the current $J_{3}$. The arrows indicate the directions of the currents $J_{1}, J_{2}$, and $J_{3}$. The multiplicity of the arrows corresponds to the number $\gamma=1,2,3$ of the current $J_{\gamma}$.

In this graph, there exists 8 cycles $c_{i}$ which are respectively (123), (134), (234), (1234), (124), (1423), (1243) and (567). A priori, there exist four independent currents $J_{k}$ with the corresponding thermodynamic forces $X_{k}$ $(k=1,2,3,4)$. In order to illustrate the case where two mesoscopic currents contribute to the same macroscopic current, we suppose that the mesoscopic transitions $1 \rightarrow 2$ and $4 \rightarrow 1$ equally contribute to the macroscopic current $J_{3}$. On the other hand, we assume that the transitions $6 \rightarrow 7$ contribute to the macroscopic current $J_{1}$ and the transitions $4 \rightarrow 2$ to the current $J_{2}$. Consequently, the macroscopic currents are given in terms of the cycle currents according to 


$$
\begin{aligned}
& J_{1}=J_{(567)} \\
& J_{2}=J_{(234)}-J_{(124)}-J_{(1243)}-J_{(1324)} \\
& J_{3}=J_{(123)}+J_{(134)}+2 J_{(124)}+2 J_{(1234)}+J_{(1243)}
\end{aligned}
$$

which allows us to read the coefficients $\rho_{k}(c)$. Using Eq. (12), the affinities of the cycles can thus be expressed in terms of the macroscopic thermodynamic forces as

$$
\begin{aligned}
& A_{(567)}=X_{1}, \quad A_{(234)}=X_{2}, \quad A_{(134)}=X_{3}, \quad A_{(123)}=X_{3}, \\
& A_{(1324)}=X_{3}-X_{2}, \quad A_{(1234)}=2 X_{3}, \quad A_{(124)}=2 X_{3}-X_{2}, \quad A_{(1243)}=X_{3}-X_{2} .
\end{aligned}
$$

Using the techniques developed in Ref. [31], we can derive a fluctuation theorem for these currents which takes the form

$$
\frac{\operatorname{Prob}\left[\left\{\frac{1}{t} \int_{0}^{t} d t^{\prime} j_{\gamma}\left(t^{\prime}\right) \in\left(\alpha_{\gamma}, \alpha_{\gamma}+d \alpha_{\gamma}\right)\right\}\right]}{\operatorname{Prob}\left[\left\{\frac{1}{t} \int_{0}^{t} d t^{\prime} j_{\gamma}\left(t^{\prime}\right) \in\left(-\alpha_{\gamma},-\alpha_{\gamma}+d \alpha_{\gamma}\right)\right\}\right]} \simeq \exp \sum_{\gamma} X_{\gamma} \alpha_{\gamma} t \quad(t \rightarrow \infty)
$$

with $\gamma=1,2,3$.

Now, we may wonder if only one of these currents obeys a single macroscopic current fluctuation theorem. According to the analysis of Subsection 4.2, we have to look at the cycles contributing to the given current. Let us consider the current $J_{3}$. In this case, we readily see from Eq. (27) and Fig. 1 that the different cycles contributing to the macroscopic current (25) present the same affinity if one sets $X_{2}=0$ regardless of $X_{1}$. In this case, we have the fluctuation theorem

$$
\frac{\operatorname{Prob}\left[\frac{1}{t} \int_{0}^{t} d t^{\prime} j_{3}\left(t^{\prime}\right) \in(\alpha, \alpha+d \alpha)\right]}{\operatorname{Prob}\left[\frac{1}{t} \int_{0}^{t} d t^{\prime} j_{3}\left(t^{\prime}\right) \in(-\alpha,-\alpha+d \alpha)\right]} \simeq \exp X_{3} \alpha t \quad(t \rightarrow \infty)
$$

which concerns the single macroscopic current $I_{3}=(1 / t) \int_{0}^{t} d t^{\prime} j_{3}\left(t^{\prime}\right)$. Indeed, the symmetry (19) is equivalent to the large-deviation property (29). The probability distribution of $(1 / t) \int_{0}^{t} d t^{\prime} j_{3}\left(t^{\prime}\right)$ is illustrated in Fig. 2 for different values of the parameters while the fluctuation relation (29) is checked in Fig. 3. In the same way, we have a fluctuation symmetry for $J_{2}$ if $X_{3}=0$, while $J_{1}$ always satisfies a fluctuation theorem. Note that the fluctuation theorem for $J_{2}$ and $J_{3}$ would hold even if the right-hand part of the graph, namely the states 6 and 7 , would be replaced by a more complex graph with more states and transitions.

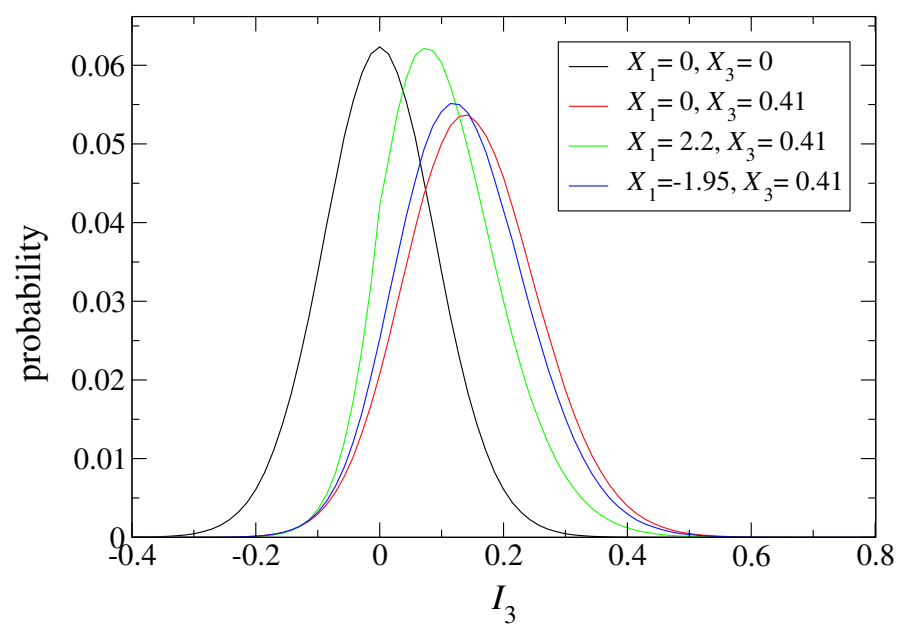

Figure 2. Probability distribution of $I_{3}=(1 / t) \int_{0}^{t} d t^{\prime} j_{3}\left(t^{\prime}\right)$ for different values of the thermodynamic forces $X_{1}, X_{3}$ at time $t=70 . X_{2}$ must be equal to zero. The parameters take the values $k_{12}=k_{23}=k_{34}=k_{56}=k_{67}=k_{78}=1.5$, $k_{21}=k_{32}=k_{43}=k_{65}=k_{76}=k_{87}=1, k_{18}=k_{81}=2$, while the other transition rates are fixed according to the thermodynamic forces $X_{\gamma}$. 


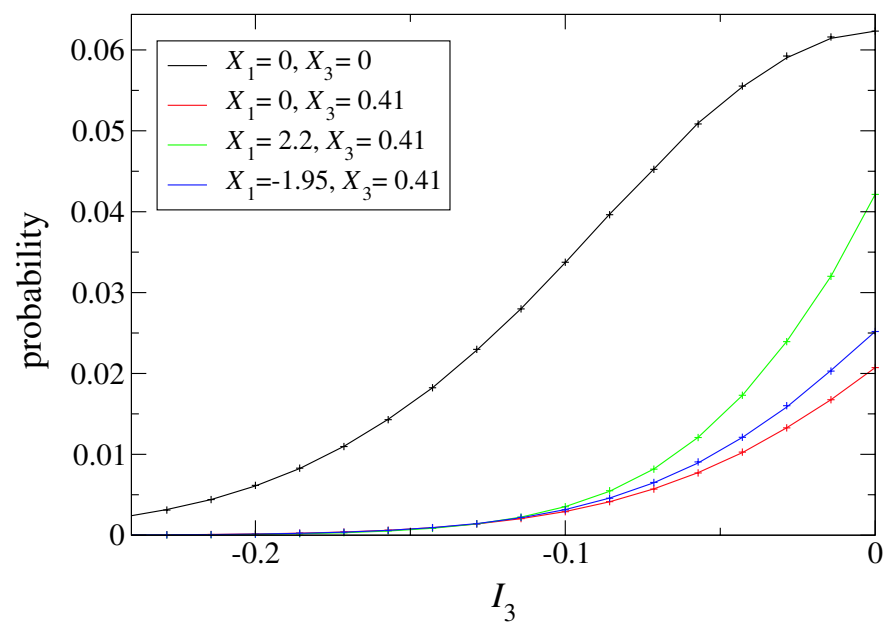

Figure 3. Negative part of the probability distribution of $I_{3}=(1 / t) \int_{0}^{t} d t^{\prime} j_{3}\left(t^{\prime}\right)$ for the same values of the thermodynamic forces $X_{\gamma}$ as in Fig. 2. In each case, the probability distribution is compared with the prediction (29) of the fluctuation theorem with the macroscopic affinity $X_{3}$ (crosses). A nice agreement is observed in all the cases.

\subsection{Chemical reaction network}

Here, we consider the following reaction network:

$$
\mathrm{R}_{1} \underset{k_{-1}}{\stackrel{k_{+1}}{\rightleftharpoons}} \mathrm{N}, \quad \mathrm{R}_{2} \underset{k_{-2}}{\stackrel{k_{+2}}{\rightleftharpoons}} \mathrm{N}, \quad \cdots \mathrm{R}_{r} \underset{k_{-r}}{\stackrel{k_{+r}}{\rightleftharpoons}} \mathrm{N}
$$

For this simple network, the transition rates are given by the mass-action law [5]

$$
\begin{aligned}
& W_{\rho}(N \mid N+1)=k_{+\rho}\left\langle R_{\rho}\right\rangle \\
& W_{\rho}(N \mid N-1)=k_{-\rho} N
\end{aligned}
$$

where $\left\langle R_{\rho}\right\rangle$ denotes the mean number of reactant molecules $\mathrm{R}_{\rho}$ with $\rho=1,2, \ldots, r$ and $N=0,1,2, \ldots$ is the fluctuating number of molecules of the intermediate species N. With these transition rates, the stationary state of the master equation (1) is given by the Poisson distribution:

with the mean value

$$
P_{\text {st }}(N)=\mathrm{e}^{-\langle N\rangle_{\text {st }}} \frac{\langle N\rangle_{\text {st }}^{N}}{N !}
$$

$$
\langle N\rangle_{\mathrm{st}}=\frac{\sum_{\rho=1}^{r} k_{+\rho}\left\langle R_{\rho}\right\rangle}{\sum_{\rho=1}^{r} k_{-\rho}}
$$

Therefore, this model has several transition mechanisms $\rho=1,2, \ldots, r$. Although it does not satisfy the network conditions of Section 3, we will show that it nevertheless does satisfy a fluctuation relation for each single macroscopic current. This is due to the particular topology of the network for these transition rates.

Here, we present the generating function of one current in order to illustrate the single macroscopic current fluctuation theorem with an analytical solution. The detailed derivation is given in the appendix. We find the following result for the fluctuations of reaction 1

$$
\begin{aligned}
Q(\lambda) & \equiv \lim _{t \rightarrow \infty}-\frac{1}{t} \ln \left\langle\mathrm{e}^{-\lambda \int_{0}^{t} j_{1}\left(t^{\prime}\right) d t^{\prime}}\right\rangle \\
& =\sum_{\rho=1}^{r} k_{+\rho}\left\langle R_{\rho}\right\rangle-\frac{\sum_{\rho=2}^{r} k_{+\rho}\left\langle R_{\rho}\right\rangle}{\left(\sum_{\rho=1}^{r} k_{-\rho}\right)\left(\sum_{\rho=2}^{r} k_{-\rho}\right)} \phi(\lambda) \phi(A-\lambda)
\end{aligned}
$$

where

$$
\phi(\lambda)=k_{-1} \mathrm{e}^{\lambda}+\sum_{\rho=2}^{r} k_{-\rho} .
$$


The driving force is here given by

$$
A=X_{1}+\ln \left(\frac{\sum_{\rho=2}^{r} k_{-\rho}}{\sum_{\rho=2}^{r} k_{-\rho} \mathrm{e}^{X_{\rho}}}\right)
$$

with the thermodynamic forces

$$
X_{\rho} \equiv \ln \frac{k_{-r} k_{+\rho}\left\langle R_{\rho}\right\rangle}{k_{-\rho} k_{+r}\left\langle R_{r}\right\rangle}
$$

and $X_{r}=0$. The asymmetry with respect to the reaction $r$ results from our choice of the dependent reaction in the network. The function (35) presents the symmetry

$$
Q(\lambda)=Q(A-\lambda)
$$

of the fluctuation theorem. Here, the driving force is not directly given by one of the thermodynamic forces $X_{\rho}$, although both are macroscopic.

\section{Conclusions}

In this paper, we derived the conditions under which a fluctuation theorem should hold for a single macroscopic current. These conditions can be expressed in terms of the cycles and transport processes occurring in the network. If the transport process is accomplished over an ensemble of cycles with the same macroscopic affinity the corresponding current will obey a fluctuation theorem with a driving force corresponding to this macroscopic affinity. These results are important for several applications as it is not always possible to keep track of all the currents present in the system. The fluctuation theorem for single currents has also important consequences as discussed in Ref. [37] in the context of flashing ratchets. It can be shown that the conditions we have here derived do no apply to such systems where a fluctuation theorem should not exist for single currents. This can already be checked in the simple model introduced in Ref. [38]. However, there could exist several systems where single macroscopic currents would satisfy a fluctuation symmetry. This is for instance the case in the simple model we constructed or for a chemical reaction network. This fluctuation relation could also be used to detect new biochemical cycles. Indeed, if a fluctuation relation is not observed for a current when its conditions of validity are satisfied, this would strongly suggests the existence of a hidden - even rare - biochemical cycle as such cycles will usually break the fluctuation symmetry of an individual current.

\section{Acknowledgements}

The authors thank Professor G. Nicolis for support and encouragement in this research. This research is financially supported by the F .N .R .S . Belgium and the "Communauté française de Belgique" (contract "Actions de Recherche Concertées" No. 04/09-312).

\section{Appendix A. Current generating function for the chemical network}

For simplicity, we consider the case of a reaction network with only two reservoirs. The general case is obtained thanks to the substitutions $k_{+2}\left\langle R_{2}\right\rangle \rightarrow \sum_{\rho=2}^{r} k_{+\rho}\left\langle R_{\rho}\right\rangle$ and $k_{-2} \rightarrow \sum_{\rho=2}^{r} k_{-\rho}$.

Let us introduce the probability $P(N, k, t)$ to have $N$ particles at time $t$ while having a signed cumulated current of $k$ particles for the reaction 1 . We can derive an evolution equation for this quantity in the following way

$$
\begin{aligned}
\frac{d P(N, k, t)}{d t} & =k_{+1}\left\langle R_{1}\right\rangle P(N-1, k-1, t)-k_{+1}\left\langle R_{1}\right\rangle P(N, k, t) \\
& +k_{-1}(N+1) P(N+1, k+1, t)-k_{-1} N P(N, k, t) \\
& +k_{+2}\left\langle R_{2}\right\rangle P(N-1, k, t)-k_{+2}\left\langle R_{2}\right\rangle P(N, k, t) \\
& +k_{-2}(N+1) P(N+1, k, t)-k_{-2} N P(N, k, t)
\end{aligned}
$$


Let us introduce the generating function of this quantity

$$
F\left(s, s_{1}, t\right)=\sum_{N=0}^{\infty} \sum_{k=-\infty}^{+\infty} s^{N} s_{1}^{k} P(N, k, t)
$$

With the knowledge of $F$, we can recover the various probabilities by developing $F$ in powers of $s$ and $s_{1}$, or equivalently by taking derivatives at $s=1$. Note that we can trace out the information we do not need by putting the corresponding variable $s$ or $s_{1}$ equal to unity. Besides, the normalisation requires that $F(1,1, t)=1, \forall t$. Since we are interested in the steady state, the initial condition will be

or

$$
P(N, k, t=0)=\mathrm{e}^{-\langle N\rangle} \frac{\langle N\rangle^{N}}{N !} \delta_{0, k}
$$

$$
F\left(s, s_{1}, t=0\right)=\mathrm{e}^{\langle N\rangle(s-1)}
$$

We can now deduce the evolution equation for $F\left(s, s_{1}, t\right)$

$$
\frac{\partial F\left(s, s_{1}, t\right)}{\partial t}=\sum_{N=0}^{\infty} \sum_{k=-\infty}^{+\infty} s^{N} s_{1}^{k} \frac{\partial P(N, k, t)}{\partial t}
$$

which, after some manipulations, becomes

$$
\begin{aligned}
\frac{\partial F\left(s, s_{1}, t\right)}{\partial t}= & {\left[k_{+1}\left\langle R_{1}\right\rangle\left(s s_{1}-1\right)+k_{+2}\left\langle R_{2}\right\rangle(s-1)\right] F\left(s, s_{1}, t\right) } \\
& +\left[k_{-1}\left(\frac{1}{s_{1}}-s\right)+k_{-2}(1-s)\right] \frac{\partial F\left(s, s_{1}, t\right)}{\partial s}
\end{aligned}
$$

We now have to solve a first-order partial differential equation with the initial condition (A.4). Using the method of characteristics to solve it, we find

$$
\begin{aligned}
F\left(s, s_{1}, t\right)= & \exp \left[t \frac{A C-B D}{D}-\frac{B}{D}-\frac{(A-B) C}{D^{2}}\left(1-\mathrm{e}^{-D t}\right)\right] \\
& \times \exp \left\{s\left[\frac{B}{D} \mathrm{e}^{-D t}+\frac{A}{D}\left(1-\mathrm{e}^{-D t}\right)\right]\right\}
\end{aligned}
$$

where

$$
\begin{aligned}
& A=k_{+1}\left\langle R_{1}\right\rangle s_{1}+k_{+2}\left\langle R_{2}\right\rangle \\
& B=k_{+1}\left\langle R_{1}\right\rangle+k_{+2}\left\langle R_{2}\right\rangle \\
& C=\frac{k_{-1}}{s_{1}}+k_{-2} \\
& D=k_{-1}+k_{-2}
\end{aligned}
$$

We notice that $\langle N\rangle=B / D$. We can check the consistency condition of the normalisation, and also that the steady state solution is recovered if $s_{1}=1$. Since we are interested in the current, we can evaluate the function $F$ at $s=1$ with $s_{1}=\mathrm{e}^{-\lambda}$ to get

$$
F\left(1, s_{1}, t\right)=\sum_{N=0}^{\infty} \sum_{k=-\infty}^{+\infty} s_{1}^{k} P(N, k, t)=\sum_{k=-\infty}^{+\infty} \mathrm{e}^{-\lambda k} P(k, t)=\left\langle\mathrm{e}^{-\lambda \int_{0}^{t} j_{1}\left(t^{\prime}\right) d t^{\prime}}\right\rangle \sim \mathrm{e}^{-Q(\lambda) t}
$$

where $P(k, t)$ is the probability of a signed cumulated current of $k$ particles for the reaction 1 , regardless the number $N$ of particles of the intermediate species $N$. In this way, we should obtain the generating function (35) of the fluctuations of reaction 1 in the long-time limit $t \rightarrow \infty$. Using the solution (A.7), we find that

$$
F\left(1, s_{1}, t\right)=\exp \left[t \frac{A C-B D}{D}-\frac{(A-B)(C-D)}{D^{2}}\left(1-\mathrm{e}^{-D t}\right)\right] \sim \mathrm{e}^{-Q(\lambda) t}
$$

In the long-time limit $t \rightarrow \infty$, the generating function (35) is thus given by

$$
Q(\lambda)=B-\frac{A C}{D}=k_{+1}\left\langle R_{1}\right\rangle+k_{+2}\left\langle R_{2}\right\rangle-\frac{1}{k_{-1}+k_{-2}}\left(k_{-1} \mathrm{e}^{\lambda}+k_{-2}\right)\left(k_{+1}\left\langle R_{1}\right\rangle \mathrm{e}^{-\lambda}+k_{+2}\left\langle R_{2}\right\rangle\right)
$$

according to the definitions (A.8)-(A.11) with $s_{1}=\mathrm{e}^{-\lambda}$. 
Finally, we obtain the expression (35) using the substitution mentioned at the beginning of this appendix and the expression (37) of the driving force.

\section{References}

[1] I. Prigogine, Introduction to Thermodynamics of Irreversible Processes (Wiley, New York, 1967).

[2] G. Nicolis and I. Prigogine, Proc. Natl. Acad. Sci. (USA) 68, 2102 (1971).

[3] G. Nicolis, J. Stat. Phys. 6, 195 (1972).

[4] G. Nicolis and J. W. Turner, Physica A 89, 326 (1977).

[5] G. Nicolis and I. Prigogine, Self-Organization in Nonequilibrium Systems (Wiley, New York, 1977).

[6] Luo Jiu-li, C. Van den Broeck, and G. Nicolis, Z. Phys. B - Condensed Matter 56, 165 (1984).

[7] J. Schnakenberg, Rev. Mod. Phys. 48, 571 (1976)

[8] H. J. Kreuzer and S. H. Payne, Theoretical Approaches to the Kinetics of Adsorption, Desorption and Reactions at Surfaces, in: M. Borowko, Computational Methods in Surface and Colloid Science (Dekker, New York, 2000) p.439.

[9] T. De Donder and P. Van Rysselberghe, Affinity (Stanford University Press, Menlo Park CA, 1936).

[10] P. Gaspard, Chaos, Scattering and Statistical Mechanics (Cambridge University Press, Cambridge UK, 1998).

[11] C. Jarzynski, Phys. Rev. Lett. 78, 2690 (1997)

[12] C. Jarzynski, J. Stat. Mech., P09005 (2004).

[13] D.-Q. Jiang, M. Qian, and M.-P. Qian, Mathematical Theory of Nonequilibrium Steady States (Springer, Berlin, 2004).

[14] D. J. Evans, E. G. D. Cohen, and G. P. Morriss, Phys. Rev. Lett. 71, 2401 (1993).

[15] D. J. Evans and D. J. Searles, Phys. Rev. E 50, 1645 (1994).

[16] G. Gallavotti and E. G. D. Cohen, Phys. Rev. Lett. 74, 2694 (1995).

[17] G. Gallavotti, Phys. Rev. Lett. 77, 4334 (1996).

[18] D. Ruelle, J. Stat. Phys. 95, 393 (1999).

[19] J. Kurchan, J. Phys. A: Math. Gen. 31, 3719 (1998).

[20] G. E. Crooks, Phys. Rev. E 60, 2721 (1999).

[21] J. L. Lebowitz and H. Spohn, J. Stat. Phys. 95, 333 (1999).

[22] C. Maes, J. Stat. Phys. 95, 367 (1999).

[23] C. Maes and K. Netočný, J. Stat. Phys. 110, 269 (2003).

[24] P. Gaspard, J. Chem. Phys. 120, 8898 (2004).

[25] U. Seifert, Phys. Rev. Lett. 95, 040602 (2005).

[26] M. Amman, R. Wilkins, E. Ben-Jacob, P. D. Maker, and R. C. Jaklevic, Phys. Rev. B 43, 1146 (1991).

[27] H. Noji, R. Yasuda, M. Yoshida, and K. Kinosita, Nature 386, 299 (1997)

[28] D. Andrieux and P. Gaspard, J. Stat. Mech.: Th. Exp. P01011 (2006).

[29] D. Andrieux and P. Gaspard, Phys. Rev. E 74, 011906 (2006).

[30] D. Andrieux and P. Gaspard, J. Chem. Phys. 121, 6167 (2004).

[31] D. Andrieux and P. Gaspard, J. Stat. Phys. 127, 107 (207).

[32] D. Andrieux and P. Gaspard, J. Stat. Mech. P02006 (2007).

[33] M.-P. Qian, C. Qian, and M. Qian, Sci. Sinica (Series A) 27, 470 (1984).

[34] T. L. Hill and O. Kedem, J. Theor. Biol. 10, 399 (1966)

[35] T. L. Hill, J. Theor. Biol. 10, 442 (1966).

[36] T. L. Hill, Thermodynamics for Chemists and Biologists (Addison-Wesley, Reading MA, 1968).

[37] W. De Roeck and C. Maes, cond-mat/0610369 (2006).

[38] C. Jarzynski and O. Mazonka, Phys. Rev. E 59, 6448 (1999). 